\title{
Subclavian Vein Cannulation Success Rate in Neonates and Children
}

\author{
Reza Aminnejad ${ }^{1}$; Seyed Sajjad Razavi ${ }^{1}$; Seyed Amir Mohajerani ${ }^{1}$; Seyed Alireza Mahdavi ${ }^{1{ }^{*}}$ \\ ${ }^{1}$ Department of Anesthesiology, Mofid Hospital, Shahid Beheshti University of Medical Sciences, Tehran, Iran \\ *Corresponding author: Seyed Alireza Mahdavi, Department of Anesthesiology, Mofid Hospital, Shahid Beheshti University of Medical Sciences, Tehran, Iran. Tel/Fax:+98-2122908383, \\ E-mail: r.aminnejad@yahoo.com
}

Received: October 27, 2014; Revised: March 13, 2015; Accepted: April 27, 2015

\begin{abstract}
Background: Central vein cannulation allows the administration of large volumes of fluids in short times and at high osmolarities for rehydration, volume replacement, chemotherapy, and parenteral nutrition. Percutaneous central venous line insertion has replaced peripheral venous cut-down as the primary mode of short-term venous access in children.

Objectives: The aim of our study was to delineate some aspects of this procedure as well as its success rate and relative risk in pediatrics. Patients and Methods: Totally, 3264 subclavian vein cannulations in neonates and children were analyzed regarding successful catheterization attempts and early complication rates after the procedure retrospectively in Mofid Hospital (Tehran, Iran).

Results: There were 1340 newborn patients (first 28 days of life) in our study population. In these newborns, only 55 cannulations failed; one patient was complicated with pneumothorax; guide wires malfunctioned in 21 cases; and first- attempt cannulation success was reported in only 981 cases. In the remaining 1924 patients, between one month and 8 years old, only 14 attempts at the cannulation of the subclavian vein failed and 1655 cases had first-attempt cannulation success.

Conclusions: The cannulation of the central vein in neonates and children in a skilled hand would be performed with great success rate and low complications.
\end{abstract}

Keywords: Subclavian Vein; Catheterization; Central Venous Line; Pediatrics; Infant, Newborn; Parenteral Nutrition

\section{Background}

Central venous (CV) lines are one of the safest and most appropriate routes of fluid administration in pediatrics and adults. Total parenteral nutrition (TPN) and central venous pressure measurements are some of its advantages. This cannulation provides a fast route to administer large amounts of intravenous fluids with high osmolarities to patients for volume compensation or TPN or during cardiopulmonary resuscitation or chemotherapy. This technique is widely used in adults but could be used unaltered in pediatrics (1). In pediatrics, there is no clearly proven superior method but the subclavian vein (SCV) cannulation has gained much attention recently. There is a $3-34 \%$ risk of side effects such as pneumothorax and infections; nevertheless, these side effects depend on age, weight, and expertise of the clinician (2). Although chest X ray, fluoroscopy, and ultrasound have been used to confirm the position of the SCV cannula in pediatrics (3), none of these modalities have actually improved the success rate of cannulation. Many of these side effects such as pneumothorax, hydrothorax, and chylothorax are found when the left SCV has been used for cannulation even when an ultrasoundassisted procedure is attempted (4). Catheter tip malposition is more often seen in the right SCV than left-sided cannulation and is not associated with patients' age (5). Although CV line insertion encounters several technical problems in pediatrics, its failure or success rate depends on the expertise of the clinician and the use of the proper technique. The success rate and other procedural aspects of CV line in pediatrics have not been discussed in details previously.

\section{Objectives}

The aim of our study was to delineate some aspects of this procedure as well as its success rate and relative risk in pediatrics.

\section{Patients and Methods}

This prospective study, conducted over a 2-year period (2010 - 2011), recruited all pediatric patients who underwent CV line insertion in Mofid Hospital (Tehran, Iran). The patients' age, sex, and other demographics were recorded. CV line was inserted through the SCV, and all the lines were inserted by one anesthesiologist, who performed the procedure in the same way. The devices were provided by the same company in all the patients, and all the side effects were recorded. The rates of first-attempt cannulation success and failure were also recorded.

The procedure was performed with the patient in the supine position and the hand adducted. The subjects were sedated prior to the procedure. After prep and drape with disinfectants, the skin was injected with local anesthetics. In smaller patients, particularly the infants under 6 months,

Copyright (C) 2015, Iranian Society of Regional Anesthesia and Pain Medicine(ISRAPM). This is an open-access article distributed under the terms of the Creative Commons Attribution-NonCommercial 4.0 International License (http://creativecommons.org/licenses/by-nc/4.0/) which permits copy and redistribute the material just in noncommercial usages, provided the original work is properly cited. 
arterial line catheters (\#G20 or \#G22) (Arrow, Germany) were employed instead of CV line catheters. In bigger children, double- or triple-lumen CV line catheters (\#G18 or \#G16) (Arrow, Germany) were used. The needle was inserted at 3-5 mm caudate to the midclavicular line under the clavicle. Then, the syringe was aspirated until dark blood jetted into it. Thereafter, the guide wire was passed to the appropriate line. If the vein was not punctured on first attempt or the guide wire encountered resistance, then the needle was pulled back to the skin and re-inserted in the cephalad direction. The tip of the catheter was subsequently inserted to be approximated at the superior vena cava. After fixation, for double check-up, control chest radiography was taken. In mechanically ventilated patients, disconnection from the ventilator was performed immediately before needle insertion so as to lessen the risk of pleural puncture and subsequent pneumothorax.

\section{Results}

The present study enrolled 3264 patients, comprised of
1526 (46\%) females and 1738 (54\%) males. The age distribution is depicted in Table 1 . The first-attempt success rate is shown in Table 1 based on age distribution. First-attempt success was achieved in 73\% of the newborns ( $0-28$ days old). Failed CV line cannulation was in 25 cases, which is depicted in Table 1 based on age distribution. The left or right subclavian CV lines are also classified based on age distribution in Table 1. Only one case in all the 3264 patients contracted pneumothorax after CV line insertion, which needed chest tube insertion. Guide-wire malfunction occurred in $7 \%$ of the cases.

In $91.5 \%$ of the patients under one month, arterial line catheters were used instead of CV line catheters due to their smaller size. None of the attempts in the patients aged over 5 years led to failure. The reasons for CV line cannulation were classified based on TPN or lack of IV line access. Most cases were cannulated due to access to a proper IV line (Table 2 ).

The success rates of cannulation in the infants and children over one month were significantly different $(\mathrm{P}=$ 0.0312) (Risk Ratio: 9.143) (Table 3).

\begin{tabular}{|c|c|c|c|c|c|c|}
\hline Age & Total & Right SCV ${ }^{\mathrm{a}}$ & Left SCV & First-Attempt Insertion & Failed Procedure & Guide-Wire Malfunction \\
\hline $0-28, d$ & 1340 & 438 & 992 & 981 & 11 & 121 \\
\hline 1-12, mo & 962 & 169 & 793 & 762 & 7 & 75 \\
\hline $1-2, y$ & 557 & 67 & 490 & 521 & 1 & 33 \\
\hline $2-3, y$ & 195 & 55 & 140 & 181 & 2 & 6 \\
\hline $3-4, y$ & 95 & 27 & 68 & 90 & 3 & 3 \\
\hline $4-5, y$ & 63 & 23 & 40 & 57 & 1 & 1 \\
\hline $5-8, y$ & 37 & 8 & 29 & 35 & 0 & 0 \\
\hline$>8, y$ & 15 & 5 & 10 & 9 & 0 & 0 \\
\hline
\end{tabular}

a Abbreviation: SCV: Subclavian Vein.

\begin{tabular}{|c|c|c|c|c|}
\hline \multirow[t]{2}{*}{ Age } & \multicolumn{2}{|c|}{ Used Catheter } & \multicolumn{2}{|c|}{ Cause of CVC } \\
\hline & Arterial Line & CV-Line & TPN & IV-Line Access \\
\hline $0-28, d$ & 1226 & 114 & 381 & 959 \\
\hline 1-12, mo & 721 & 241 & 267 & 695 \\
\hline $1-2, y$ & 330 & 227 & 138 & 419 \\
\hline $2-3, y$ & 124 & 71 & 48 & 147 \\
\hline $3-4, y$ & 30 & 65 & 69 & 26 \\
\hline $4-5, y$ & 12 & 51 & 50 & 13 \\
\hline $5-8, y$ & 7 & 30 & 28 & 9 \\
\hline$>8, y$ & 0 & 15 & 11 & 4 \\
\hline
\end{tabular}

a Abbreviations: CVC: Central Vein Cannulation, CV-Line: Central Venous Line, IV: Intravenous, TPN: Total Parenteral Nutrition. 
Table 3. Comparison of the Success Rates in Placing the Catheter in the Newborns or Infants ${ }^{\text {a,b }}$

\begin{tabular}{lccc}
\hline Variables & Failure Rate & Success Rate & Total \\
\hline Age group & & & \\
\hline Newborn & 4.8 & 95.2 & $84(30)$ \\
$\begin{array}{l}\text { Infancy and } \\
\text { pediatrics }\end{array}$ & 0.5 & 99.5 & $192(70)$ \\
Total & 2 & 98 & $276(100)$ \\
\hline
\end{tabular}

a Fisher's Exact Test: P value = 0.0312; Confidence Interval 95\% =1.037 80.622; Risk Ratio $=9.143$

$\mathrm{b}$ Values are presented as \% or No. (\%).

\section{Discussion}

$\mathrm{CV}$ catheter cannulation could be a hurdle to clinicians and anesthesiologists in the pediatric group due to the anatomical and technical difficulties and the fragility of patients. The SCV cannulation success rate has been reported up to $78.8 \%$ in infants younger than 6 months, as opposed to up to $96 \%$ in pediatrics older than 6 months (6). Citak et al. (7) showed a 95\% success rate of the SCV cannulation in their study. The total success rate in our study was $98 \%$, but there is no doubt that the two subsets of patients have differences.

Regarding the side effects of the SCV cannulation, our study depicted the incidence of these side effects in detail. One of the major side effects of this technique is the probability of pneumothorax following cannulation. Nonetheless, only one case out of our 3264 cases had pneumothorax, which underlines the low probability of this side effect. This patient had bullae emphysema in his lung as the underlying disease. Another side effect of the technique is infection or primary catheter sepsis (8), but we did not see any positive culture from our cannulations.
In conclusion, $\mathrm{CV}$ cannulation in pediatrics could be a challenging procedure and as such requires high levels of expertise if the possible side effects are to be avoided. This procedure should be performed with smaller size cannulae in newborns and infants younger than 6 months of age.

\section{Authors' Contributions}

Seyed Sajjad Razavi suggested the study design; Reza Aminnejad performed data collection, data analysis, and interpretation; Seyed Alireza Mahdavi performed critical revision and manuscript approval; and Seyed Amir Mohajerani performed manuscript drafting.

\section{References}

1. Haas NA. Clinical review: vascular access for fluid infusion in children. Crit Care. 2004;8(6):478-84.

2. de Jonge RC, Polderman KH, Gemke RJ. Central venous catheter use in the pediatric patient: mechanical and infectious complications. Pediatr Crit Care Med. 2005;6(3):329-39.

3. Motoyama EK, Davis PJ. Chapter 18. Smith's Anesthesia for Infants and Children. 7 ed. Philadelphia: Elsevier Health Sciences; 2006.

4. Froehlich CD, Rigby MR, Rosenberg ES, Li R, Roerig PL, Easley KA, et al. Ultrasound-guided central venous catheter placement decreases complications and decreases placement attempts compared with the landmark technique in patients in a pediatric intensive care unit. Crit Care Med. 2009;37(3):1090-6.

5. Casado-Flores J, Valdivielso-Serna A, Perez-Jurado L, Pozo-Roman J, Monleon-Luque M, Garcia-Perez J, et al. Subclavian vein catheterization in critically ill children: analysis of 322 cannulations. Intensive Care Med.1991;17(6):350-4.

6. Finck C, Smith S, Jackson R, Wagner C. Percutaneous subclavian central venous catheterization in children younger than one year of age. Am Surg. 2002;68(4):401-4.

7. Citak A, Karabocuoglu M, Ucsel R, Uzel N. Central venous catheters in pediatric patients-subclavian venous approach as the first choice. Pediatr Int. 2002;44(1):83-6.

8. Eichelberger MR, Rous PG, Hoelzer DJ, Garcia VF, Koop CE. Percutaneous subclavian venous catheters in neonates and children. $J$ Pediatr Surg. 1981;16(4 Suppl 1):547-53. 